\title{
Testing soft tissue radiodensity parameters interplay with age and self-reported physical activity
}

\author{
Marco Recenti (1), ${ }^{\dagger}$ Carlo Ricciardi $(1,2),{ }^{\dagger}$, Kyle Edmunds (1), Deborah Jacob (1), \\ Monica Gambacorta (3), Paolo Gargiulo $(1,4)$ \\ (1) Institute for Biomedical and Neural Engineering, Reykjavík University, Reykjavík, Iceland; \\ (2) Department of Electrical Engineering and Information Technology, University of Naples \\ 'Federico II', Naples, Italy; (3) Umberto I Hospital, ASL Salerno, Nocera Inferiore, Italy; (4) \\ Department of Science, Landspítali, Reykjavík, Iceland. \\ ${ }^{\dagger}$ Marco Recenti and Carlo Ricciardi contributed equally as co-first author \\ This article is distributed under the terms of the Creative Commons Attribution Noncommercial License (CC BY-NC 4.0) which permits \\ any noncommercial use, distribution, and reproduction in any medium, provided the original author(s) and source are credited.
}

\begin{abstract}
Aging well is directly associated to a healthy lifestyle. The focus of this paper is to relate individual wellness with medical image features. Non-linear trimodal regression analysis (NTRA) is a novel method that models the radiodensitometric distributions of $\mathrm{x}$-ray computed tomography (CT) cross-sections. It generates 11 patient-specific parameters that describe the quality and quantity of muscle, fat, and connective tissues. In this research, the relationship of these 11 NTRA parameters with age, physical activity, and lifestyle is investigated in the 3,157 elderly volunteers AGES-I dataset. First, univariate statistical analyses were performed, and subjects were grouped by age and self-reported past (youth-midlife) and present (within 12 months of the survey) physical activity to ascertain which parameters were the most influential. Then, machine learning (ML) analyses were conducted to classify patients using NTRA parameters as input features for three ML algorithms. ML is also used to classify a Lifestyle index using the age groups. This classification analysis yielded robust results with the lifestyle index underlying the relevant differences of the soft tissues between age groups, especially in fat and connective tissue. Univariate statistical models suggested that NTRA parameters may be susceptible to age and differences between past and present physical activity levels. Moreover, for both age and physical activity, lean muscle parameters expressed more significant variation than fat and connective tissues.
\end{abstract}

Key Words: CT Scan; lifestyle; physical activity, machine learning, soft tissues.

Eur J Transl Myol 31 (3): 9929, 2021 doi: 10.4081/ejtm.2021.9929

The loss of skeletal muscle mass and function is acknowledged in the literature as an independent risk factor for impairments and deleterious health effects in the elderly population. ${ }^{1-3}$ This phenomenon, widely known as sarcopenia, has been directly linked to fracture risk, bone fragility, disability, and injurious falls. ${ }^{4}$ Agingrelated pathologies and comorbidities, such as diabetes, ${ }^{5}$ hypertension, ${ }^{6}$ and cardiovascular disease, ${ }^{7}$ have been likewise associated with sarcopenia and decreased muscle strength. Moderate to vigorous physical activity is protective against the adverse effects of sarcopenia. ${ }^{8}$ Different exercise training approaches not only improve the overall quality of life, ${ }^{9}$ but also preserve the health of muscle fibers that otherwise weaken with age. ${ }^{10,11}$ Muscle mass and strength can be a predictor factor of incident mobility limitation. ${ }^{12}$ Physical activity levels are predicted in the literature mainly by follow-up studies: physical activity levels in adulthood are related to the performances and sports activities in adolescence. ${ }^{13}$ Muscle mass and strength can also be a predictor of longevity when considering body mass index as a primary indicator; ${ }^{14}$ however, the concomitant assessment of age using quantitative assessment modalities of lean muscle or other soft tissue remains absent in the literature. Instead, machine learning (ML) and deep learning approaches have been used for this purpose, using diffusion tensor imaging, ${ }^{15}$ and structural brain MRI, ${ }^{16}$ as initial classification features.

Our team recently developed a novel soft tissue assessment method based upon cross-sectional computed tomography (CT) images, known as non-linear trimodal regression analysis (NTRA), which models radiodensitometric distributions to generate 11 patient- 


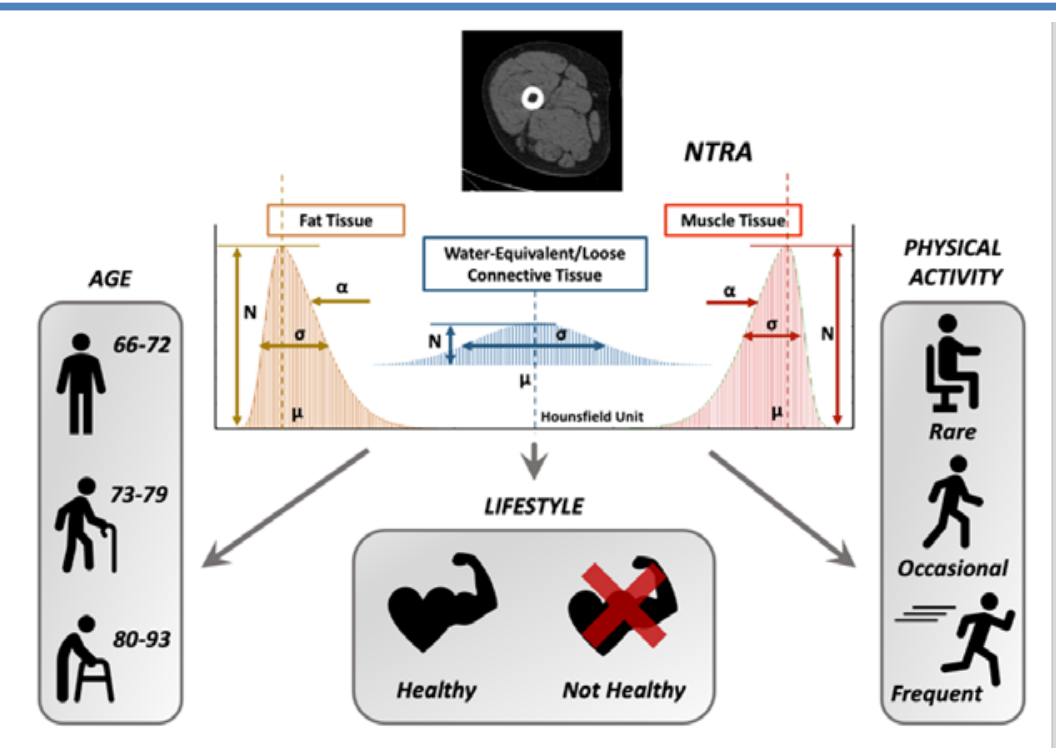

Fig 1. Workflow of the present study: 11 soft tissue radiodensity parameters extracted from a mid-thigh CT-Scan are used to conduct statistical analysis and classify age, self-reported physical activity, and a lifestyle index

specific soft tissue parameters. ${ }^{17,18}$ This method was motivated by identifying an alternative approach to study changes in soft tissue changes mass and quality as a quantitative construct for sarcopenia in the elderly. In this regard, NTRA parameters have been shown to be predictive factors for lower extremity function, cardiovascular pathophysiology, body mass index, hypertension, and diabetes mellitus. ${ }^{19-24}$ However, exploring the sensitivity of these parameters to age and physical activity using univariate statistical analyses remained unperformed. Further analysis is performed using the lifestyle index presented by Recenti et al. ${ }^{21}$ based on both physical activity and smoking status to study the radiodensitometry differences between groups of age. Fig. 1 shows the study workflow, where we report the use of NTRA parameters to conduct statistical analyses to classify age, physical activity levels, and lifestyle index.

\section{Materials and Methods}

\section{AGES-Reykjavik Database}

The database used in the present study was an initial cross-sectional subset of the AGES-Reykjavík study known as AGES-I. This database contains data from 3,157 healthy elderly subjects from 66-93 years of age (mean: 74.87) who participated in a multimodal series of biometric measurements. ${ }^{25}$ The Icelandic National Bioethics Committee gave the ethical approval for this work (RU Code of Ethics, cf. Paragraph 3 in Article 2 of the Higher Education Institution Act no. 63/2006). Informed consent was obtained from all participants. Subjects received CT scans and were also surveyed for additional information, such as age and physical activity levels. Due to twenty missing surveys, our total sample size for physical activity analyses was $n=3.137$ subjects.
For this study, the AGES-I database was divided into three groups according to three age classes: the first group is composed of people between 66 and 72 years of age (total of 1.176 subjects), while the second group age interval was 73-79 years (1.371 subjects), and the third was from $80-83$ years (610 subjects). Five categories of self-reported physical activity frequency were defined in the AGES-I database for both past (youth or midlife) and present levels (within 12 months of the survey): 'never', 'rarely', 'occasionally', 'moderate', and 'high'. Based on this self-reported information, the database was again divided into three classes. The "Frequent" class included people that performed moderate or high levels of activity in the past or present (1.462 subjects), while the "Occasional" class included subjects with occasional activity frequency in the past or present (488 subjects). All remaining subjects were placed in the "Rare - No Activity" class (1.187 subjects). The Lifestyle Index, part of a more extensive binary-tree division of the AGES-I database, as previously published in Recenti $\mathrm{M}$, et al. (2020), ${ }^{21}$ divides the cohort into healthy (469) and not healthy (2668) individuals according to their selfreported physical activity frequency and smoking status. The Mid-Thigh CT-scan acquisition protocol, described in detail in Edmunds $\mathrm{KJ}$ et al. (2016) gives radiodensitometric distributions from cross-sectional pixel matrices defined using transformed radiodensity values across the range for soft tissue, between -200 and 200 Hounsfield Units (HU). ${ }^{17}$

\section{Non-Linear Trimodal Regression}

The NTRA method, described for the first time in 2018 by Edmunds et al., ${ }^{17}$ interprets each $\mathrm{HU}$ distribution as a quasi-probability density function defined by Gaussian radiodensity distributions of three soft tissue types: lean 
muscle [41 to $200 \mathrm{HU}$ ], fat [-200 to $-10 \mathrm{HU}$ ], and loose connective tissue and atrophic muscle [ -9 to $40 \mathrm{HU}]$. Eq. 1 defines this trimodal quasi-probability density function where $\mathrm{N}$ is the distribution amplitude, $\mu$ is the location in $\mathrm{HU}$, and $\sigma$ is the distribution width (Figure 1). The skewness term, $\alpha$, considers the inwardly sloping asymmetries, and it is not considered for the central connective tissue distribution. This definition allows for the subject-specific fitting of HU distribution curves via standard error minimization at each radio-absorption bin using a generalized reduced gradient algorithm. This results in the extraction of 11 subject-specific NTRA parameters.

$$
\begin{array}{r}
\sum_{i=1}^{3} \varphi\left(x, N_{i}, \mu_{i}, \mu_{i}, \alpha_{i}\right)= \\
\sum_{1}^{3} \frac{N_{i}}{\sigma_{i} \sqrt{2 \pi}} e^{-\frac{\left(x-\mu_{i}\right)^{2}}{2 \sigma_{i}^{2}}} \operatorname{erfc}\left(\frac{\alpha_{i}\left(x-\mu_{i}\right)}{\sigma_{i} \sqrt{2}}\right)
\end{array}
$$

Machine Learning: tools, algorithms, evaluation metrics Knime analytics platform has been employed in this study to perform ML analyses for both regression and classification, as already performed in biomedical literature. $^{26-30}$ Three popular tree-based algorithms were employed: random forests (RF), ADA-boosting (ADA$\mathrm{B})$, and gradient boosting (GB) combined with 10-fold cross-validation. The Synthetic Minority Oversampling Technique (SMOTE) is also performed. SMOTE was implemented to increase the quality of the classification, oversampling the minority classes. Key evaluation metrics were considered to evaluate the present regression results: Coefficient of Determination $\left(\mathrm{R}^{2}\right)$, Mean Absolute Error (MAE), Mean Squared Error (MSE), and Root Mean Squared Error (RMSE). For ML classification analysis, Accuracy, Sensitivity, and Specificity were used as evaluation metrics.

\section{Results}

\section{Age: Statistical Analysis}

After assessing the non-normality of NTRA parameters, the dataset was divided into three subgroups according to age, and a one-way Kruskal Wallis test was performed. The mean and the standard deviations of age for each group are as follows: Group 1: $70.06 \pm 1.61$ years; Group 2: $75.66 \pm 1.86$ years; and Group 3: $82.35 \pm 2.39$ years. This assessment shows that all the NTRA parameters excluding the location of the connective tissue resulted in statistically significant groupwise differences.

Statistically significant differences were found in two out of three parameters in connective tissue, compared to four of four in both muscle and fat. After this Kruskal Wallis test, a posthoc test was performed: here, the differences in each parameter are computed according to age by couples of groups. Several differences were captured through the posthoc test, excluding the amplitude of fat tissue. Muscle was the tissue with the greatest number of statistically significant differences among all three age groups, followed by fat tissue. Fat skewness, connective width, and muscle amplitude, location, and skewness distinguished all three age groups.

\section{Physical Activity: Statistical Analysis}

Next, NTRA parameters were investigated according to physical activity using the same statistical analysis workflow. A one-way Kruskal Wallis test is performed. Subjects were divided into three activity level classes, "Rare - No Activity", "Occasional", and "Frequent" (respectively Groups 1, 2, and 3). Overall, 7 out of 11 parameters were statistically significant. Statistically significant differences were found in one out of three NTRA parameters in connective tissue, four out of four in muscle, and two out of three in fat. After the Kruskal Wallis test, a posthoc test was analogously performed. Several differences were captured through this second posthoc test. Muscle was again the tissue with the greatest number of statistically significant differences among all three groups, followed by fat. No parameter distinguished all three groups, but connective location, muscle width and skewness, and fat amplitude, location, and width were all able to distinguish subjects by activity level.

\section{Machine Learning for Age and Physical Activity}

ML regression models were next assembled, first focusing on the classification of subject age using NTRA parameters as initial features. Only RF and GB algorithms are reported in this analysis, as ADA-B results were consistently poor compared to these other two algorithms. Age regression was performed by considering the three age groups separately: from 66-72 years of age with 1,176 subjects, 73-79 years with 1,371 , and 80-93 years with $610 . \mathrm{R}^{2}$ results were generally weak, indicating the low variance captured by the NTRA-based model. On the contrary, results obtained from the analysis of the errors were much more indicative of a minimum error in predicting an integer, such as the age of an individual. A minimum MAE of 1.377 was found with RF classification in the first age group, where the minimum MSE was likewise only 2.55. These errors were intermediate in size in the middle range, with an MAE of about 1.5 and an MSE of 3.5. In the last age group, the numbers of subjects decreased while the errors increased, reaching an MAE of 1.8 and an MSE of 6.4 with GB classification. The results obtained from the classification of the three age classes and the physical activity classes were not particularly robust; the accuracy for age classification never eclipsed $60 \%$, with the highest value in the RF model. In physical activity, accuracy reached $60 \%$, again with the RF model yielding the best results.

\section{Lifestyle Index: Machine Learning and NTRA analysis}

According to the three age divisions, the classification of the healthy and not healthy groups gives the best results in terms of accuracy, always reaching more than 79 with 
Table 1. Metrics for lifestyle index classification

\begin{tabular}{|l|l|l|l|l|}
\hline $\begin{array}{l}\text { Age } \\
\text { Group }\end{array}$ & Alg. & Sens. & Spec. & Acc. \\
\hline $\mathbf{6 6 - 7 2}$ & RF & 90.3 & 82.3 & 86.3 \\
\hline $\mathbf{6 6 - 7 2}$ & GB & 84.6 & 73.7 & 79.1 \\
\hline $\mathbf{6 6 - 7 2}$ & ADA-B & 86.8 & 74.6 & 80.7 \\
\hline $\mathbf{7 3 - 7 9}$ & RF & 91.5 & 84.8 & 88.1 \\
\hline $\mathbf{7 3 - 7 9}$ & GB & 87.3 & 72.9 & 80.1 \\
\hline $\mathbf{7 3 - 7 9}$ & ADA-B & 90.2 & 81.8 & 86.0 \\
\hline $\mathbf{8 0 - 9 3}$ & RF & 96.2 & 88.7 & 92.5 \\
\hline $\mathbf{8 0 - 9 3}$ & GB & 91.7 & 83.5 & 87.6 \\
\hline $\mathbf{8 0 - 9 3}$ & ADA-B & 88.1 & 78.3 & 83.2 \\
\hline
\end{tabular}

a maximum of 92.5 with RF algorithm for the group of the oldest subjects (Table 1 ). RF always gives the best accuracy results higher than 86 and the best sensitivity, consistently higher than 90 with a maximum of 96.2. Table 2. A-B-C shows the average values of the 11 NTRA parameters for healthy and not healthy subjects divided by age groups. The most significant difference can be seen in the fat, muscle, and connective amplitude $(\mathrm{N})$ and a big difference in the location $(\mu)$ of the connective tissue, especially between healthy and not healthy people in each age group.

\section{Discussion}

ML results prove that the CT-scan extracted NTRA parameters can classify with a very high accuracy the Lifestyle Index using the three age groups. The obtained results could be helpful for patient subject to a hip prosthesis, ${ }^{31-34}$ in particular in relation to the prevention of the femur fracture risk. ${ }^{35,36}$ These results do not only strengthen the NTRA value to predict significant comorbidities like diabetes, hypertension, and cardiac diseases, ${ }^{19,24}$ but also confirm the importance of this imaging approach underlying the differences between healthy and not healthy subjects from a radiodensitometric point of view especially in the muscle and connective tissues as specified in Table 2, A,B,C. Again, as already underlined in the previous NTRA analysis with the AGES database, ${ }^{19,24}$ the connective tissue assumes high importance and shows relevant differences between age groups. NTRA parameters are also sensitive to variation in subject age, and muscle parameters particularly experience the most significant variation compared to fat and connective tissues. Regarding physical activity, NTRA parameters are strongly sensitive to differences in past and present physical activity levels. Muscle parameters again experience the most remarkable variation compared to fat and connective tissues. The radiodensitometric profiles experienced a fewer change in people who reported frequent physical activity in their past or present life. However, these data were not strengthened by the results from ML analyses at the opposite of the Lifestyle Index
Table 2. A. NTRA FAT parameters average for healty $(H)$ and not healthy $(\mathrm{NH})$ age groups.

\begin{tabular}{|c|c|c|c|c|}
\hline $\begin{array}{c}\text { Age } \\
\text { Group }\end{array}$ & n_fat & $\mu$ fat & $\sigma \_$fat & $\alpha \_$fat \\
\hline $66-72 \mathrm{H}$ & 67,41 & $-117,71$ & 7,59 & $-2,20$ \\
\hline 66-72 nH & 63,98 & $-117,75$ & 8,10 & $-2,33$ \\
\hline 73-79 H & 62,96 & $-117,88$ & 7,83 & $-2,34$ \\
\hline 73-79 nH & 60,62 & $-117,79$ & 8,39 & $-2,56$ \\
\hline $80-93$ H & 52,63 & $-118,31$ & 11,07 & $-3,81$ \\
\hline 80-93 nH & 59,85 & $-117,98$ & 8,22 & $-2,60$ \\
\hline
\end{tabular}

Table 2. B. NTRA muscle tissue parameters average for healty $(\mathrm{H})$ and not healthy $(\mathrm{NH})$ age groups.

\begin{tabular}{|c|c|c|c|c|}
\hline $\begin{array}{c}\text { Age } \\
\text { Group }\end{array}$ & n_musc & $\mu$ _musc & $\sigma$ musc & $\alpha$ _musc \\
\hline $66-72 \mathrm{H}$ & 80,41 & 62,44 & 8,13 & 2,59 \\
\hline 66-72 nH & 82,65 & 62,04 & 8,54 & 2,77 \\
\hline 73-79 H & 77,83 & 61,68 & 8,58 & 2,81 \\
\hline 73-79 nH & 77,82 & 61,36 & 8,65 & 2,83 \\
\hline $80-93$ H & 70,26 & 60,19 & 8,16 & 2,72 \\
\hline $80-93 \mathrm{nH}$ & 70,30 & 60,29 & 8,93 & 3,03 \\
\hline
\end{tabular}

Table 2. C. NTRA connective tissue parameters average for healty $(\mathrm{H})$ and not healthy $(\mathrm{NH})$ age groups.

\begin{tabular}{|c|c|c|c|}
\hline $\begin{array}{c}\text { Age } \\
\text { Group }\end{array}$ & n_conn & $\mu_{-}$conn & o_conn \\
\hline $66-72 \mathrm{H}$ & 40,69 & $-28,07$ & 26,50 \\
\hline 66-72 nH & 43,00 & $-23,06$ & 25,76 \\
\hline 73-79 H & 40,89 & $-28,37$ & 24,96 \\
\hline 73-79 nH & 41,90 & $-22,73$ & 24,92 \\
\hline $80-93$ Н & 38,72 & $-17,71$ & 23,37 \\
\hline 80-93 nH & 39,64 & $-26,30$ & 24,13 \\
\hline
\end{tabular}

classification. Error rates were meager in age regression, mainly for the lower two age groups. This does not mean that NTRA parameters are not necessarily sensitive to changes in age or the quality of physical activity, which would need to be assessed using prospective or longitudinal study designs; simply, the present $\mathrm{ML}$ models were not able to confirm the significant differences obtained from statistical analyses in the age and physical activity prediction.

Nevertheless, the univariate statistical analyses show that in-particular, fat and connective tissues are sensitive to age and self-reported physical activity in the elderly. As for the machine learning approach, other types of nontree-based algorithms could also have been tested. However, given that tree-based algorithms gave excellent results in previous NTRA applications and in the 
Lifestyle Index prediction, it is likely that the use of other algorithms would not have improved these results. This notion necessitates future comparative analyses focused on the diagnostic value of the NTRA methodology using longitudinal data. This could be performed by comparing the sensitivity to age and physical activity of NTRA parameters with standard CT analyses, such as total, fat, and lean muscle cross-sectional areas and/or average cross-sectional radiodensity. A possible future evolution of the study could be to extend the NTRA technology combined with ML not only to sarcopenia in the elderly population, but also to motor handicapped individuals: the radiodensitometry distribution of fat, lean muscle and connective tissue could be predictive of the potential physical activity and other pathologies that affect these subjects as it was for the AGES population

\section{List of acronyms}

ADA-B - AdaBoosting

AGES - Age Gene/Environment Susceptibility Study

CT - Computed Tomography

GB - Gradient-Boosting

HU - Hounsfield Unit

MAE - Mean Absolute Error

ML - Machine Learning

MSE - Mean Squared Error

NTRA - Nonlinear Trimodal Regression Analysis

$\mathrm{R}^{2}$ - Coefficient of Determination

RF - Random Forest

RMSE - Root Mean Squared Error

SMOTE - Synthetic Minority Oversampling Technique

\section{Authors contributions}

MR and CR performed the calculations. KJE contributed with the NTRA development and implementation. DJ reviewed and helped with the drafting. MG contributed with the medical knowledge. PG supervised and coordinated the whole study. All the authors contributed to editing and revising the draft.

\section{Acknowledgments}

The authors wish to thank the University Hospital Landspitali in Reykjavik for their infrastructural support and Hjartavernd for the AGES-Reykjavik dataset.

\section{Funding}

This research did not receive any specific grant from funding agencies in the public, commercial, or not-forprofit sectors.

\section{Data Availability Statement}

The AGES dataset cannot be made publicly available: requests for these data may be sent to the AGESReykjavik Study Executive Committee, contact: Ms. Camilla Kristjansdottir, camilla@hjarta.is

\section{Conflict of Interest}

The authors declare they have no financial, personal, or other conflicts of interest.

\section{Ethical Publication Statement}

We confirm that we have read the Journal's position on issues involved in ethical publication and affirm that this report is consistent with those guidelines.

\section{Corresponding Author}

Prof. Paolo Gargiulo, Reykjavik University, Institute of Biomedical and Neural Engineering, Menntavegi 1,102 Reykjavik, Iceland ORCID iD: 0000-002-5049-4817

E-mail: paologar@landspitali.is

E-mails and ORCID iD of co-authors

Marco Recenti: marco18@ru.is

ORCID iD: 0000-0001-9440-8434

Carlo Ricciardi: carloricciardi.93@gmail.com

ORCID iD: 0000-0001-7290-6432

Kyle Edmunds: kyle14@ru.is

ORCID iD: 0000-0002-6591-4116

Deborah Jacob: deborah20@ru.is

ORCID iD: 0000-0003-3507-8003

Monica Gambacorta: m.gambacorta@aslsalerno.it

ORCID iD: 0000-0002-0532-4464

\section{References}

1. Barberi L, Scicchitano BM, Musaro A. Molecular and cellular mechanisms of muscle aging and sarcopenia and effects of electrical stimulation in seniors. Eur J Transl Myol. 2015 Aug 25;25(4):2316. doi: 10.4081/ejtm.2015.5227.

2. Kalyani RR, Corriere M, Ferrucci L. Age-related and disease-related muscle loss: the effect of diabetes, obesity, and other diseases. Lancet Diabetes Endocrinol. 2014 Oct;2(10):819-29. doi: 10.1016/S2213-8587(14)70034-8. Epub 2014 Mar 6.

3. Goodpaster BH, Carlson CL, Visser M, Kelley DE, Scherzinger A, Harris TB, Stamm E, Newman AB. Attenuation of skeletal muscle and strength in the elderly: The Health ABC Study. J Appl Physiol (1985). 2001 Jun;90(6):2157-65. doi: 10.1152/jappl.2001.90.6.2157

4. Marzetti E, Calvani R, Tosato M, Cesari M, Di Bari M, Cherubini A, Collamati A, D'Angelo E, Pahor M, Bernabei R, Landi F; SPRINTT Consortium. Sarcopenia: an overview. Aging Clin Exp Res. 2017 Feb;29(1):11-17. doi: 10.1007/s40520-016-0704-5. Epub 2017 Feb 2.

5. Newman AB, Kupelian V, Visser M, Simonsick E, Goodpaster B, Nevitt M, Kritchevsky SB, Tylavsky FA, Rubin SM, Harris TB; Health ABC Study Investigators. Sarcopenia: alternative definitions and associations with lower extremity function. J Am Geriatr Soc. 2003 Nov;51(11):1602-9. doi: 10.1046/j.1532-5415.2003.51534.x.

6. Brooks SV, Faulkner JA. Skeletal muscle weakness in old age: underlying mechanisms. Med Sci Sports Exerc. 1994 Apr;26(4):432-9. 
7. Han P, Yu H, Ma Y, Kang L, Fu L, Jia L, Chen X, Yu X, Hou L, Wang L, Zhang W, Yin H, Niu K, Guo $\mathrm{Q}$. The increased risk of sarcopenia in patients with cardiovascular risk factors in Suburb-Dwelling older Chinese using the AWGS definition. Sci Rep. 2017 Aug 29;7(1):9592. doi: 10.1038/s41598-017-084888.

8. Cruz-Jentoft AJ, Landi F, Schneider SM, Zúñiga C, Arai H, Boirie Y, Chen LK, Fielding RA, Martin FC, Michel JP, Sieber C, Stout JR, Studenski SA, Vellas B, Woo J, Zamboni M, Cederholm T. Prevalence of and interventions for sarcopenia in ageing adults: a systematic review. Report of the International Sarcopenia Initiative (EWGSOP and IWGS). Age Ageing. 2014 Nov;43(6):748-59. doi: 10.1093/ageing/afu115. Epub 2014 Sep 21.

9. Landi F, Marzetti E, Martone AM, Bernabei R, Onder G. Exercise as a remedy for sarcopenia. Curr Opin Clin Nutr Metab Care. 2014 Jan;17(1):25-31. doi: 10.1097/MCO.0000000000000018.

10. Šarabon N, Smajla D, Kozinc Ž, Kern H. Speedpower based training in the elderly and its potential for daily movement function enhancement. Eur J Transl Myol. 2020 Apr 1;30(1):8898. doi: 10.4081/ejtm.2019.8898.

11. Cvecka J, Tirpakova V, Sedliak M, Kern H, Mayr W, Hamar D. Physical Activity in Elderly. Eur J Transl Myol. 2015 Aug 25;25(4):249-52. doi: 10.4081/ejtm.2015.5280.

12. Visser M, Goodpaster BH, Kritchevsky SB, Newman AB, Nevitt M, Rubin SM, Simonsick EM, Harris TB. Muscle mass, muscle strength, and muscle fat infiltration as predictors of incident mobility limitations in well-functioning older persons. J Gerontol A Biol Sci Med Sci. 2005 Mar;60(3):324-33. doi: 10.1093/gerona/60.3.324.

13. Glenmark B, Hedberg G, Jansson E. Prediction of physical activity level in adulthood by physical characteristics, physical performance and physical activity in adolescence: an 11-year follow-up study. Eur J Appl Physiol Occup Physiol. 1994;69(6):5308. doi: 10.1007/BF00239871.

14. Srikanthan P, Karlamangla AS. Muscle mass index as a predictor of longevity in older adults. Am J Med. 2014 Jun;127(6):547-53. doi: 10.1016/j.amjmed. 2014.02.007. Epub 2014 Feb 18.

15. Mwangi B, Hasan KM, Soares JC. Prediction of individual subject's age across the human lifespan using diffusion tensor imaging: a machine learning approach. Neuroimage. 2013 Jul 15;75:58-67. doi: 10.1016/j.neuroimage.2013.02.055. Epub 2013 Mar 14.

16. Jonsson BA, Bjornsdottir G, Thorgeirsson TE, Ellingsen LM, Walters GB, Gudbjartsson DF, Stefansson H, Stefansson K, Ulfarsson MO. Brain age prediction using deep learning uncovers associated sequence variants. Nat Commun. 2019
Nov 27;10(1):5409. doi: 10.1038/s41467-01913163-9.

17. Edmunds KJ, Árnadóttir Í, Gíslason MK, Carraro U, Gargiulo P. Nonlinear Trimodal Regression Analysis of Radiodensitometric Distributions to Quantify Sarcopenic and Sequelae Muscle Degeneration. Comput Math Methods Med. 2016;2016:8932950. doi: 10.1155/2016/8932950. Epub 2016 Dec 27.

18. Edmunds K, Gíslason M, Sigurðsson S, Guðnason V, Harris T, Carraro U, Gargiulo P. Advanced quantitative methods in correlating sarcopenic muscle degeneration with lower extremity function biometrics and comorbidities. PLoS One. 2018 Mar 7;13(3):e0193241. doi: 10.1371/journal.pone. 0193241.

19. Ricciardi C, Edmunds KJ, Recenti M, Sigurdsson S, Gudnason V, Carraro U, Gargiulo P. Assessing cardiovascular risks from a mid-thigh CT image: a tree-based machine learning approach using radiodensitometric distributions. Sci Rep. 2020 Feb 18;10(1):2863. doi: 10.1038/s41598-020-59873-9.

20. Recenti M, Ricciardi C, Monet A, Jacob D, Ramos J, Gislason MK, Edmunds KJ, Carraro U, Gargiulo P. Predicting body mass index and isometric leg strength using soft tissue distributions from computed tomography scans. Health Technol. 2020 Nov 4; 1-11. doi: https://doi.org/10.1007/s12553020-00498-3.

21. Recenti M, Ricciardi C, Edmunds KJ, Gislason MK, Sigurdsson S, Carraro U, Gargiulo P. Healthy Aging Within an Image: Using Muscle Radiodensitometry and Lifestyle Factors to Predict Diabetes and Hypertension. IEEE J Biomed Health Inform. 2021 Jun;25(6):2103-2112. doi: 10.1109/JBHI.2020. 3044158. Epub 2021 Jun 3.

22. Recenti M, Ricciardi C, Edmunds K, Gislason MK, Gargiulo P. Machine learning predictive system based upon radiodensitometric distributions from mid-thigh CT images. Eur J Transl Myol. 2020 Apr 1;30(1):8892. doi: 10.4081/ejtm.2019.8892.

23. Recenti M, Ricciardi C, Gìslason MJ, Edmunds KJ, Carraro U, Gargiulo P. Machine learning algorithms predict body mass index using nonlinear trimodal regression analysis from computed tomography scans. Mediterranean Conference on Medical and Biological Engineering and Computing. 2019 Sept 25; 839-846. Springer, Cham. doi:10.1007/978-3030-31635-8_100

24. Recenti M, Gìslason MK, Edmunds KJ, Gargiulo P. Aging Health Behind an Image: Quantifying Sarcopenia and Associated Risk Factors from Advanced CT Analysis and Machine Learning Technologies. In International Symposium on Computer Methods in Biomechanics and Biomedical Engineering. 2019 Aug;188-197. Springer, Cham. doi:10.1007/978-3-030-431952_15. 
25. Harris TB, Launer LJ, Eiriksdottir G, Kjartansson O, Jonsson PV, Sigurdsson G, Thorgeirsson G, Aspelund T, Garcia ME, Cotch MF, Hoffman HJ, Gudnason V. Age, Gene/Environment Susceptibility-Reykjavik Study: multidisciplinary applied phenomics. Am J Epidemiol. 2007 May 1;165(9):1076-87. doi: 10.1093/aje/kwk115. Epub 2007 Mar 10.

26. Stanzione A, Ricciardi C, Cuocolo R, Romeo V, Petrone J, Sarnataro M, Mainenti PP, Improta G, De Rosa F, Insabato L, Brunetti A, Maurea S. MRI Radiomics for the Prediction of Fuhrman Grade in Clear Cell Renal Cell Carcinoma: a Machine Learning Exploratory Study. J Digit Imaging. 2020 Aug;33(4):879-887. doi: 10.1007/s10278-02000336-y.

27. Ricciardi C, Cuocolo R, Cesarelli G, Ugga L, Improta G, Solari D, Romeo V, Guadagno E, Cavallo LM, Cesarelli, M. Distinguishing functional from non-functional pituitary macroadenomas with a machine learning analysis. Paper presented at the IFMBE Proceedings. 2019 Sep 25; 76 1822-1829. doi:10.1007/978-3-030-31635-8_221.

28. Ricciardi C, Improta G, Amato F, Cesarelli G, Romano M. Classifying the type of delivery from cardiotocographic signals: A machine learning approach. Comput Methods Programs Biomed. 2020 Nov;196:105712. doi: 10.1016/j.cmpb.2020. 105712. Epub 2020 Aug 18.

29. Ricciardi C, Valente AS, Edmund K, Cantoni V, Green R, Fiorillo A, Picone I, Santini S, Cesarelli M. Linear discriminant analysis and principal component analysis to predict coronary artery disease. Health Informatics J. 2020 Sep;26(3):21812192. doi: 10.1177/1460458219899210. Epub 2020 Jan 23.

30. Recenti M, Ricciardi C, Aubonnet R, Picone I, Jacob D, Svansson HÁR, Agnarsdóttir S, Karlsson GH, Baeringsdóttir V, Petersen H, Gargiulo P. Toward Predicting Motion Sickness Using Virtual Reality and a Moving Platform Assessing Brain, Muscles, and Heart Signals. Front Bioeng Biotechnol. 2021 Apr 1;9:635661. doi: 10.3389/fbioe.2021.635661.

31. Ricciardi C, Jónsson H Jr, Jacob D, Improta G, Recenti M, Gíslason MK, Cesarelli G, Esposito L, Minutolo V, Bifulco P, Gargiulo P. Improving Prosthetic Selection and Predicting BMD from Biometric Measurements in Patients Receiving Total Hip Arthroplasty. Diagnostics (Basel). 2020 Oct 14;10(10):815. doi: 10.3390/diagnostics10100815.

32. Gargiulo P, Edmunds KJ, Gíslason MK, Latour C, Hermannsson D, Esposito L, Bifulco P, Cesarelli M, Fraldi M, Cristofolini L, Jónsson H Jr. Patientspecific mobility assessment to monitor recovery after total hip arthroplasty. Proc Inst Mech Eng H. 2018 Oct;232(10):1048-1059. doi: 10.1177/ 0954411918797971. Epub 2018 Sep 7.

33. Fraldi M, Esposito L, Perrella G, Cutolo A, Cowin SC. Topological optimization in hip prosthesis design. Biomech Model Mechanobiol. 2010 Aug;9(4):389-402. doi: 10.1007/s10237-009-01830 .

34. Esposito L, Bifulco P, Gargiulo P, Fraldi M. Singularity-free finite element model of bone through automated voxel-based reconstruction. Comput Methods Biomech Biomed Engin. 2016 Feb;19(3):257-262. doi: 10.1080/10255842. 2015.1014347. Epub 2015 Feb 27.

35. Esposito L, Bifulco P, Gargiulo P, Gíslason MK, Cesarelli M, Iuppariello L, Jónsson H, Cutolo A, Fraldi M. Towards a patient-specific estimation of intra-operative femoral fracture risk. Comput Methods Biomech Biomed Engin. 2018 Sep;21(12):663-672. doi: 10.1080/10255842. 2018.1508570. Epub 2018 Oct 27.

36. Esposito, L, Minutolo, V, Gargiulo, P, Jonsson, H Jr, Gislason, MK, Fraldi, M. Towards an App to Estimate Patient-Specific Perioperative Femur Fracture Risk. Appl Sci. 2020 Sep; 10(18) 6409. doi:10.3390/app10186409.

Submission: June 21, 2021 Accepted for publication: July 7, 2021 